Shared care

\section{Shared care: step down or step up?}

\section{E J Maher, D Millar}

\section{Shared care arrangements between hospitals and primary care for the management of patients with cancer must be seen as a step up from "usual" care in general practice rather than a step down from hospital practice.}

$\mathrm{F}$ ormal "shared care" in cancer remains controversial. It is promoted as offering patients care closer to home while, at the same time, reducing the burden on specialist services. Primary care practitioners are divided on the issue, some viewing shared care as enhancing their practice and others as another example of hospitals offloading work onto an already overloaded primary care sector. There is some evidence to suggest that primary care based follow up alone is acceptable to patients and provides similar outcomes to specialist follow up in breast cancer, ${ }^{2}$ although this is disputed by others who cite patient preference ${ }^{3}$ or difficulties with detection of recurrence ${ }^{4}$ as arguments for continued specialist review. There is also increased interest in models of selfmanagement, with patients accessing the most appropriate healthcare professionals to meet their needs. ${ }^{5}$ What is clear is that much follow up activity is based on historical practice rather than evidence.

The study from Aarhus University by Nielsen et $a l^{6}$ in this issue of QSHC suggests a positive effect on patient evaluation of the healthcare system following the introduction of a shared care programme for newly diagnosed cancer patients. This enhancement of care is achieved in part by the provision by specialists of comprehensive information about the individual, their cancer and its management to the GPs, and also by providing communication channels and clear guidance to patients themselves about whom to contact and in what circumstances. This information transfer across the interface between secondary and primary care allows GPs and their healthcare teams to offer optimal care and support to cancer patients and their families and enhances relationships and communication between the different care agencies. It follows therefore that such information ought to be available as a matter of good practice whether or not formal shared care arrangements are in place.

Some guidance ${ }^{7}$ exists for best practice in terms of information transfer on discharge from hospital. Unfortunately, even the basic minimum data are often unavailable to primary care on discharge, and significant delays often occur before it is received. This seriously hampers the ability of GPs to manage cases effectively. Continuing efforts are required to rectify this persisting problem.

A report produced jointly by the UK Royal College of General Practitioners and the Faculty of Oncology, Royal College of Radiologists ${ }^{8}$ suggested that, given the lack of evidence of effectiveness for structured follow up arrangements, as many as $70 \%$ of patients might safely stop hospital based follow up without detriment to the desired outcomes of early recognition of recurrence of new disease, psychosocial support, and opportunities for audit, research and training. It states that "the effectiveness of these arrangements would depend on fully informing the patient, gaining their complete compliance and the prompt referral and assessment on discovery of new signs of symptoms of disease" and "there appears room for further development of both primary care based follow up and some shared care arrangements in those patients willing to accept these forms of care".

Any development of formal shared care arrangements in cancer should involve discussion with all those involved and, most importantly, with the patients and carers themselves. Rigorous evaluation of such schemes is required and further research needed to identify which groups of patients with which cancers are best managed in this way. Such collaborative work can only improve intersectoral understanding of roles and responsibilities, so long as provision of the patients' needs is kept central. Such schemes will only work if, after appropriate training, GPs with an interest in this work see it as enhancing patient care, improving job satisfaction, and associated with appropriate remuneration. It must be seen as a step up from "usual" care in general practice rather than a step down from hospital practice.

Qual Saf Health Care 2003;12:242

\section{Authors' affiliations}

E J Maher, D Millar, Mount Vernon Hospital, Northwood, Middlesex HA6 2RN, UK

Correspondence to: Dr E J Maher, Mount Vernon Hospital, Northwood, Middlesex HA6 2RN, UK; jane.maher@mvh-limc.org

\section{REFERENCES}

1 Grunfeld E, Mant D, Yudkin P, et al. Routine follow up of breast cancer in primary care: randomised trial. BM 1996:313:665-9.

2 Grunfeld E, Fitzpatrick R, Mant D, et al. Comparison of breast cancer patient satisfaction with follow-up in primary care versus specialist care: results from a randomized controlled trial. Br J Gen Pract 1999;49:705-10.

3 Maher J, Bradburn J, Adewuyi-Dalton R. Patients prefer specialist follow up. BM 1995:311:54

4 Dixon J, Norman B. Most recurrences after breast conservation are detected by regular hospital visits. BM 1996;313:1548.

5 Bradburn J, Maher EJ, Adewuyi-Dalton R, et al. Developing clinical trial protocols: the use of patient focus groups. Psycho-Oncology 1995;4:107-12.

6 Nielsen JD, Palshof T, Mainz J, et al. Randomised controlled trial of a shared care programme for newly referred cancer patients: bridging the gap between general practice and hospital. Qual Saf Health Care 2003:12:263-72.

7 Scottish Intercollegiate Guidelines Network. Interface between the hospital and the community: the immediate discharge document. Edinburgh: Scottish Intercollegiate Guidelines Network, 1996.

8 Royal College of General Practitioners and Faculty of Clinical Oncology, Royal College of Radiologists. Cancer care follow-up: an evolving service. London: Royal College of General Practitioners and Faculty of Clinical Oncology, Royal College of Radiologists, 2000 


\section{"Doing prescribing": high hopes and unexplored beliefs}

\section{G Elwyn, J Braspenning}

A novel approach to assessing prescribing behaviour involving the views of both patients and doctors, combined with an independent view of "appropriateness", provides a sophisticated approach to the act of prescribing.

M ost consultations between doctors and patients involve transactions that pivot on exchanging a piece of paper on which is inscribed the name of a potion. The prescription is literally an "order" that should be followed by the patient, reified by a pharmaceutical intermediary who enacts the alchemy signified by this most symbolic of documents

This description uses rather mystical terms perhaps, but it does so in order to point up the often forgotten ritualistic psychotherapeutic elements of this activity. "Doing prescribing" interactions are replete with decisions, many of which involve conflicts, ambivalences and reassurances. Patients will be asking: "is my problem worthy of attention?"; "will it resolve on its own?"; "will the doctor think I'm wasting time?"; "is it worth taking medicine given the opportunity cost, direct cost, possible side effects and interference with 'natural' defences?". On the professional side it is similarly complex: "how confident am I that this set of symptoms fits the presumed diagnosis?"; "is it worth intervening with a medication (similar cost issues)?"; "I don't want to 'disappoint" this patient by 'offering' nothing"; "will they be satisfied if I offer them reassurance without a prescription?". On the dark side of this equation is the use of prescriptions to terminate transactions-practitioners who use medication as the token to signal that their transaction is at an end: "take this three times a day and be gone". Two sets of preferences need to come together, yet the outcome can be evaluated along many dimensions including patient compliance, a professional's job satisfaction, and good clinical practice.

Studies on prescribing behaviour focus on counting volume, cost, type of drug, or adherence to guidelines, ${ }^{1}$ but there have been few attempts to get under the surface of the prescribing process. ${ }^{23}$ Few studies have tried to model the preferences of the negotiators and to make clear how they affect the outcome, although there are exceptions. ${ }^{4}$ In this issue of QSHC a study by Britten and colleagues ${ }^{6}$ makes an excellent contribution by combining the patient and prescriber perspectives and also adding a pharmacological dimension. Prescriptions were classified into any combination of the following three categories: unwanted (patient), unnecessary (doctor), and inappropriate (independent view based on analyses of medical record and drug prescribed). The authors have highlighted the study's weaknesses, yet the work stands out because of its preparedness to develop a more sophisticated approach to the act of prescribing. They reported many interesting findings, such as the confirmation (again) that patients declare lower expectations for prescribing than the rate that actually occurs $(42 \%$ compared with 65\%). Another interesting finding was the paradox that nearly a quarter of unnecessary prescribing (according to doctors' views) was "wanted" by the patient (patient expectations). Adherence to these prescriptions was problematic (not unexpectedly).

However, despite the novel approach, we would like to raise two issues: (1) the difficult concept of appropriateness and (2) the placing of the doctor in the position of being a judge of his or her own behaviour and the research method chosen for this. Appropriateness is a "slippery customer" and is dependent on differing viewpoints. Using carefully agreed criterion definitions can help but, if more than one criterion is selected or if different stakeholders judge the same criterion, a weighting problem is introduced. The drug prescribed can be appropriate according to the expectations of the patient but inappropriate according to a necessity defined by the doctor, or appropriate or otherwise according to an external judge using a pharmacological perspective. Britten et al address the issue by using the criterion of "appropriate prescribing" which they seem to value above the others and consider it to be more "objective". Maybe this is correct, and we should place patient or clinician views secondary to external judgements. However, this is debatable and the study makes it clear that we should set this weighting problem on our research agendas. More data will be needed to determine the contribution of each criterion to predict the degree of adherence, for instance.
Secondly, we were surprised to notice that the doctors questioned approximately one out of five of their decisions in terms of the prescribing necessity. We know that there is a temptation for window dressing when individuals are questioned about their own behaviour. It is therefore possible that unnecessary prescribing decisions are even more commonplace. Perhaps we should develop tools to support doctors at earlier points along the decision pathway. Decision support software might offer a way both to support and investigate these decisions, if ways were found of making such technologies fit into consulting and prescribing behaviours.

Parts of these prescribing interactions will remain difficult, if not impossible, to quantify. The theoretical field of decision making has suggested another qualitative method called "thinking aloud". This method has been applied to doctors, ${ }^{7}$ but combining different perspectives will pose new challenges. As this study reveals, prescribing is as much about the negotiation by two people of emotionally coloured belief systems as it is about the use of rational pharmacological interventions. There is undoubtedly more room to investigate the quality of "doing prescribing".

Qual Saf Health Care 2003;12:243

\section{Authors' affiliations}

G Elwyn, Primary Care Research Group, University of Wales Swansea Clinical School, Swansea SA2 8PP, UK

J Braspenning, Centre for Quality of Care Research, Universities of Nijmegen and Maastricht, 6500 HB Nijmegen, The Netherlands

Correspondence to: Professor G Elwyn, Primary Care Research Group, University of Wales Swansea Clinical School, Swansea SA2 8PP, UK; g.elwyn@swansea.ac.uk

\section{REFERENCES}

1 van Staa TP, Cooper C, Leufkens HG, et al. The use of inhaled corticosteroids in the United Kingdom and the Netherlands. Respir Med 2003:9:578-85.

2 Makoul G, Arntson P, Schofield T. Health promotion in primary care: physician-patient communication and decision-making about prescription medications. Soc Sci Med 1995;41:1241-54

3 Butler CC, Rollnick S, Pill R, et al. Understanding the culture of prescribing: qualitative study of general practitioners' and patients' perceptions of antibiotics for sore throats. BM 1998;317:637-42.

4 Cockburn J, Pit S. Prescribing behaviour in clinical practice: patients' expectations and the doctors' perceptions of patients' expectations-a questionnaire study. BM 1997:315:520-3.

5 Britten N, Stevenson FA, Barry CA, et al. Misunderstandings in prescribing decisions in general practice: qualitative study. BM 2000;320:484-8.

6 Britten N, Jenkins L, Barber N, et al. Developing a measure for the appropriateness of prescribing in general practice. Qual Saf Health Care 2003;12:246-50.

7 Denig P, Witteman CL, Schouten HW. Scope and nature of prescribing decisions made by general practitioners. Qual Saf Health Care 2002; $11: 137-43$. 
semantics. They criticise health professionals for using the term "preeclampsia" to describe the scenario of mild hypertension and "+" proteinuria

\section{Management of hypertension in pregnancy in rural areas}

\section{Maresh}

If we are to develop national guidelines, they must encompass all regions of the country and make allowances for rural areas.

$\mathrm{H}$ ypertension in pregnancy remains one of the leading causes of maternal deaths with approximately 1 in 100000 women in the UK dying from complications associated with it. ${ }^{1}$ In other parts of the world the figure is higher. Maternal and perinatal morbidity is relatively common in the $0.5-1 \%$ of women who have severe hypertension, with eclamptic fits occurring in about 1 in 2000 pregnancies. ${ }^{2}$ However, hypertension in pregnancy is also a common complication with up to $10 \%$ of pregnant women having a significant rise in blood pressure in the third trimester. Furthermore, complications may develop rapidly with a minimal rise in blood pressure. Encouraging pregnant women to have as much antenatal care as possible in the community therefore produces challenges for health professionals. They have to be able to predict the small number who may develop severe complications so that they can be appropriately managed to minimise the risk of maternal and perinatal morbidity and mortality.

Such challenges are even more marked in rural areas with poor routes of communication. Studies in other medical specialties have shown a tendency for more patients to be referred to central hospitals from rural areas because of anxieties that complications may occur in non-ideal settings. Local guidelines have often been produced to assist in the process. In Scotland a guideline for the management of non-proteinuric hypertension in pregnancy has been compiled using the methodology of the Scottish Intercollegiate Guidelines Network $(\mathrm{SIGN}) .^{3}$ Guidelines produced in this way are highly regarded, having been subjected to a rigorous development process. They are widely circulated and are sent to all general practitioners providing maternity care in Scotland. However, despite the fact that hypertension in pregnancy is a common condition, there is little evidence based on randomised controlled studies so most of the recommendations are graded as level $\mathrm{B}$ or $\mathrm{C}$.

In this issue of QSHC Tucker et $a l^{4}$ report a study in which they investigated the management of hypertensive conditions in pregnancy to see whether this guideline was used in practice. A questionnaire approach was used involving two clinical scenarios. Only minimal information was givenincluding maternal age, gestation, blood pressure recording, and the degree of proteinuria. The study found that most of the general practitioners and midwives working in rural settings did not follow the guideline and tended to overrefer or arrange emergency hospital admissions.

\section{WHY ARE THE GUIDELINES NOT BEING FOLLOWED?}

One of the problems in translating guidelines into practice is that clinicians may not want to follow them precisely when the evidence base is not very robust. An example from the study by Tucker et al is the recommendation in the guideline of a diastolic BP cut off of $90 \mathrm{~mm} \mathrm{Hg}$; some clinicians feel that an incremental rise in diastolic $\mathrm{BP}$ of more than $25 \mathrm{~mm} \mathrm{Hg}$ during pregnancy is significant, and this may occur in young women with a diastolic BP of less than $90 \mathrm{~mm} \mathrm{Hg}$. Another example is the controversy in obstetrics as to whether to use the Korotkoff phase IV (muffling) or $\mathrm{V}$ (disappearance) of the pulse as a measure of the diastolic blood pressure. A further problem arises when a key measurement on which management is based is somewhat subjective. In the study by Tucker et al one of the two scenarios described a woman with "+" proteinuria according to the dipstick test. If she had had " ++ " proteinuria the management would have been different. Bearing in mind the subjectivity of interpreting the colour differences between "+" and " ++ " on urine dipstick tests for proteinuria, one can perhaps begin to understand why the health professionals tended to err on the side of caution in their management of the patient.

A further point concerning the use of guidelines is whether an appropriate measure of compliance is used. In this study one could take issue with the authors for devoting a whole table to because the guideline refers to it as "mild hypertension". This is despite the fact that the guideline admits that multiple names are used for the condition.

It is also important to consider whether the guideline is actually appropriate for a particular population. Tucker et al looked at the uptake of guidelines in rural settings and, in particular, analysed the results by the distance of the practice from a specialist hospital. In keeping with other studies, they found that referral or admission rather than community monitoring were more likely to be advised in cases further from hospital. However, those working in isolated rural communities are being asked to take additional responsibility for a wide range of conditions, not just hypertension in pregnancy. The authors quote one general practitioner who commented on the barrage of guidelines, the infrequency of certain conditions, the individual approach to patients, and the difficulties of management in remote communities. Perhaps he/she should have been writing this commentary as it summarises so many of the problems. Patient expectation is increasing and there has to be equity of health care. Is it correct that we should have the same admission rate for a particular hypertensive problem in a woman who lives on an island with no hospital facility and one who lives almost next to a large maternity unit? How many admissions, unnecessary in retrospect, equate to one emergency helicopter flight? Who should make these decisions? If we are to develop national guidelines, they must encompass all regions of the country and make allowances for rural areas. Although the SIGN guideline development methodology encompasses user representatives, perhaps more consideration needs to be given to ensuring additional consultation from practitioners and patients from rural communities. The sense of local ownership is one of the main determinants as to whether or not a guideline is followed.

However, the real clinical issue is improving the support for our isolated health professionals. The authors hit the nail on the head at the end of their paper when they talk about developing "stronger partnerships between specialist centres and primary care professionals". Piles of guidelines-even if they are readily available on the web-are not a complete substitute for individualised discussions with an experienced obstetrician. With increasing centralisation of maternity care and expansion of the number of consultant obstetricians, all referral units should have an experienced obstetrician readily available to 
advise, with no need to rely on relatively inexperienced trainees. Methods of communication may obviously include the telephone and facsimile. However, medicine should be moving forward with communication technologies and ensuring that newer methods are readily available for isolated communities. Diagnostic images are transferable electronically and video consultation links should be available. There is a cost but, if we are to have equity of care, there is a price to pay. Providing quality continuing education for isolated practitioners does mean they need to spend more time on education and less on direct patient care; again there is a cost, but improving standards needs investment. Education is particularly important for those practitioners who have never (or not recently) been used to a particular style of management, such as that being proposed in this guideline. Being sent a guideline is not necessarily enough to instil the confidence needed to take on the additional responsibility.

Qual Saf Health Care 2003;0:244-245

n..................

Authors' affiliations

M Maresh, Consultant Obstetrician, St Mary's Hospital for Women and Children, Manchester M13 OJH, UK; michael.maresh@cmmc.nhs.uk
REFERENCES

1 Report on Confidential Enquiries into Maternal Deaths in the United Kingdom 1997-1999. London: The Stationery Office, 2002

2 Douglas KA, Redman CWG. Eclampsia in the United Kingdom. BM 1994;309:1395400.

3 Scottish Obstetrics Guidelines and Audit Project. The management of mild non-proteinuric hypertension. Scottish Programme for Clinical Effectiveness in Reproductive Health (SPCERH) Publication No 2. Aberdeen: SPCERH, 1997. http://www.show.scot.nhs.uk/sign/ guidelines/sogap/sogap2.html.

4 Tucker JS, Farmer J, Stimpson P. Guidelines and management of mild hypertensive conditions in pregnancy in rural general practices in Scotland: issues of

appropriateness and access. Qual Saf Health Care 2003;12:286-90. 